\title{
Manipulating exciton fine structure in quantum dots with a lateral electric field
}

\author{
B. D. Gerardot, ${ }^{\text {a) }}$ S. Seidl, P. A. Dalgarno, and R. J. Warburton \\ School of Engineering and Physical Sciences, Heriot-Watt University, Edinburgh EH14 4AS, \\ United Kingdom \\ D. Granados and J. M. Garcia \\ Instituto de Microelectronica de Madrid, CNM (CSIC), Isaac Newton 8, PTM, 28760 Tres Cantos, \\ Madrid, Spain \\ K. Kowalik and O. Krebs \\ CNRS-Laboratoire de Photonique et Nanostructures, Route de Nozay, 91460 Marcoussis, France \\ K. Karrai \\ Center for NanoScience, LMU, Geschwister-Scholl-Platz, 1, 80539 Munich, Germany
}

A. Badolato and P. M. Petroff

Materials Department, University of California, Santa Barbara, California 93106

(Received 28 August 2006; accepted 11 December 2006; published online 22 January 2007)

\begin{abstract}
The fine structure of the neutral exciton in a single self-assembled InGaAs quantum dot is investigated under the effect of a lateral electric field. Stark shifts up to $1.5 \mathrm{meV}$, an increase in linewidth, and a decrease in photoluminescence intensity were observed due to the electric field. The authors show that the lateral electric field strongly affects the exciton fine-structure splitting due to active manipulation of the single particle wave functions. Remarkably, the splitting can be tuned over large values and through zero. (C) 2007 American Institute of Physics.
\end{abstract}

[DOI: 10.1063/1.2431758]

There is currently great interest in producing entangled photons on demand for applications in quantum information processing. ${ }^{1}$ One proposal which spurred much research is using radiative biexciton-exciton cascade in semiconductor quantum dots (QDs) to produce pairs of polarization entangled photons. ${ }^{2}$ In an idealized QD, the bright exciton states $(M= \pm 1)$ are degenerate. In this case the two decay paths from the biexciton to the vacuum state via the intermediate single exciton are indistinguishable in energy; thus photons emitted in the radiative cascade are polarization entangled. However, in practice, the rotational symmetry of a self-assembled QD is broken and the electron-hole exchange interaction mixes the bright exciton states into a nondegenerate doublet [referred to as a fine-structure splitting (FSS)]. This leads to an energetically distinguishable recombination path for the biexciton-exciton cascade. Polarization correlations are observed in the linear basis but polarization entanglement is destroyed due to the FSS. ${ }^{3,4}$ For photons to be polarization entangled using this scheme, the requirement that the FSS be less than the homogeneous linewidth must be met. The FSS is typically $10-100 \mu \mathrm{eV}$, while the homogeneous linewidth of self-assembled InGaAs QDs is $\sim 1 \mu \mathrm{eV} .^{5}$

Techniques used to actively tune the FSS include an inplane electric ${ }^{6}$ or magnetic ${ }^{7}$ field and an in situ uniaxial stress. ${ }^{8}$ Also, QDs which are smaller due to the growth process ${ }^{9}$ or subsequent annealing ${ }^{10}$ have a smaller FSS. Unfortunately, such QDs are higher in energy and the QD photons become difficult to distinguish from those produced in the wetting layer. Recently, polarization entangled photons have been reported from specific QDs with energetically overlapping bright exciton states ${ }^{11}$ and initially nondegenerate states tuned via a magnetic field. ${ }^{12}$ However, a robust

\footnotetext{
${ }^{a)}$ Electronic mail: b.d.Gerardot@hw.ac.uk
}

approach that would allow one to actively tune the FSS from a large value to zero for each QD is still necessary to realize an event ready entangled photon pair source. To this end we further explore the effect of a lateral electric field on the FSS.

There are three basic characteristics of an exciton in a lateral electric field attributed to the quantum confined Stark effect, as has been investigated for quantum wells ${ }^{13}$ and QDs: ${ }^{14}$ a redshift in recombination energy, decreased oscillator strength, and an increase in nonradiative carrier tunneling probability. Additionally, electric fields also affect the FSS of a QD, ${ }^{6}$ and while a thorough understanding is still lacking the expectation of such an effect is motivated by the following. The long-range component of the exchange interaction greatly affects the FSS and is largely determined by the electron-hole overlap. ${ }^{15}$ Self-assembled QDs which are elongated along the $[1-10]$ direction impart an asymmetry onto the electron and hole wave functions. Therefore, an inplane electric field changes the single particle wave functions, thus altering the electron-hole overlap and affecting the FSS. While previous results demonstrated the ability to tune the FSS, ${ }^{6}$ we show here a much stronger relative effect such that the FSS can be tuned over a large range $(\sim 100 \mu \mathrm{eV})$ compared to the initial FSS. Furthermore, the degeneracy of the bright exciton is restored.

Our samples consist of a single layer of undoped selfassembled InAs/GaAs QDs grown by molecular beam epitaxy ( $p$-type background doping $\sim 10^{15} \mathrm{~cm}^{-3}$ ). Two CrNiAu gate electrodes, each $200 \mu \mathrm{m}$ wide and separated by a $15 \mu \mathrm{m}$ channel, were deposited using conventional photolithography. The gate fingers were aligned parallel to the [1-10] direction of the GaAs wafer. The Schottky contacts are used to induce an electric field in the plane of the QDs, which are located $130 \mathrm{~nm}$ below the surface. However, due to the roughness of the gate fingers $(\sim 3 \mu \mathrm{m})$, the exact di- 

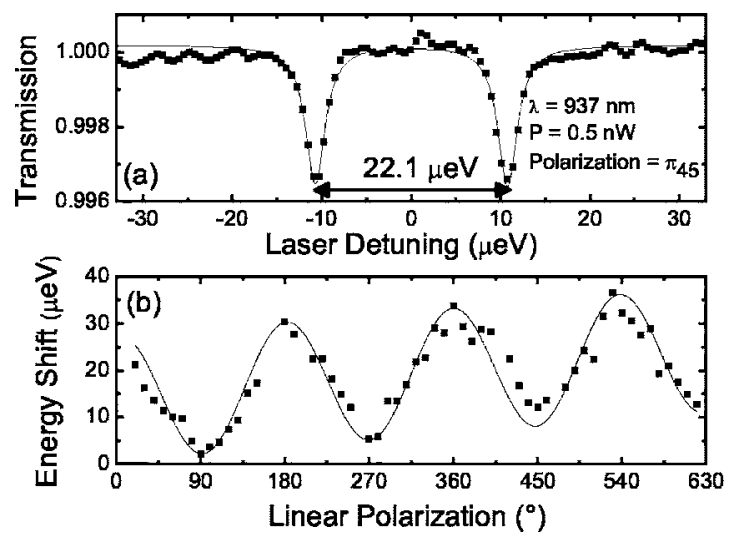

FIG. 1. FSS for the same quantum dot measured using (a) laser spectroscopy and (b) polarization dependent PL. For the laser spectroscopy, the splitting of the two peaks fit with Lorentzian lines is $22.1 \mu \mathrm{eV}$. For PL, the solid line fit for the energy shift due to polarization is a sine curve with an amplitude (i.e., FSS) of $26.6 \pm 3.4 \mu \mathrm{eV}$. Additionally, there is a small drift in the PL energy likely caused by a small temperature change in the spectrometer.

rection of the electric field relative to the crystallographic axis for each QD is not precisely known. Photoluminescence (PL) was performed using an $850 \mathrm{~nm}$ excitation laser. The spectral resolution was measured to be $\sim 33 \mu \mathrm{eV}$ [full width at half maximum (FWHM)] using a narrow band laser. The FWHM diameter of the laser spot on the sample surface is $\sim 400 \mathrm{~nm}$ due to the use of a hemispherical glass $(n=2.0)$ solid immersion lens. By measuring the PL polarization dependence we identify neutral excitons through the observation of the FSS. The doublet can be resolved with an accuracy of $\sim 5 \mu \mathrm{eV}$ by using a Lorentzian curve-fitting procedure. ${ }^{6,7}$ The accuracy of this technique was verified by comparing the doublet in PL to a FSS measurement on the same QD using transmission spectroscopy (Fig. 1), which has sub- $\mu \mathrm{eV}$ resolution. ${ }^{5}$ This comparison was made on a sample which allowed for a vertical electric field as presently required in our transmission experiment.

The PL from several QDs at different spatial positions was measured as a function of bias. In contrast to previous reports, ${ }^{14}$ for our device QDs in the center of the channel show no bias dependence, signifying that the voltage drop entirely occurs in the depletion region near the gates. By placing a detector below the sample, we can measure the incident laser power in transmission. The transmission power will decrease as the laser moves on top of the opaque $\mathrm{Cr}$ $\mathrm{NiAu}$ contact. The ratio of the laser spot on top of the Schottky gate provides a measurement of the distance of the laser spot center from the gate. In this way, we estimate the lateral depletion width to be $\sim 500 \mathrm{~nm}$ for the device. Figure 2 shows PL from a neutral exciton as a function of

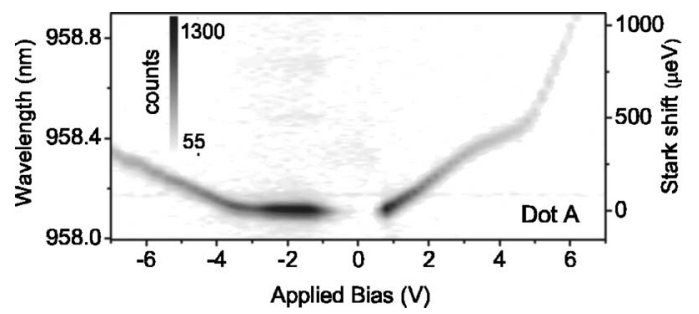

FIG. 2. Low temperature (4 K) PL of a neutral exciton as a function of applied bias from a QD located in the depletion region of the device. The PL quenches from $\sim+1.0$ to $-1.0 \mathrm{~V}$ due to a charging event, and at larger biases the exciton energy changes due to the Stark effect.

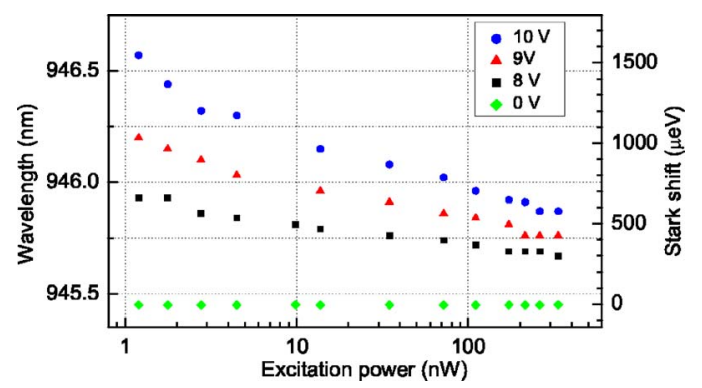

FIG. 3. (Color online) Effect of carrier screening in the device for different applied biases. At high excitation powers, the Stark effect is strongly reduced due to space-charge accumulation in the device. At low powers, Stark shifts of $\sim 1.5 \mathrm{meV}$ are observed for an applied bias of $10 \mathrm{~V}$.

applied bias for a QD located $\sim 200 \mathrm{~nm}$ from the gate. Several features are observed in the spectra. The first is the PL of the neutral exciton quenches abruptly at $\pm 1 \mathrm{~V}$ which we interpret as a charging event. PL lines shifted in energy appear in the spectra between $\pm 1 \mathrm{~V}$ (not shown), but because of the relatively high QD density ( $\sim 50$ QDs $\mu \mathrm{m}^{-2}$ ) it is difficult to assign different lines to the same dot unambiguously.

The second feature of note is the large red-shift, $\sim 1 \mathrm{mev}$ for this QD, with applied bias. A dipole in an electric field displays a Stark shift with quadratic field dependence: $E=E_{0}-p F+\beta F^{2}$, where $E$ is the energy, $F$ the electric field, $p$ the permanent dipole moment, and $\beta$ the polarizability. In our device $F$ is highly dependent on QD position and laser power (see below), so essentially the QD itself is a local in situ electric field probe. To estimate $F$ based on the Stark shift observed by PL, we assume a rotationally symmetric (in-plane) parabolic confinement potential. This leads to zero dipole moment in the $x-y$ plane. We have characterized similar QDs in other samples under the influence of vertical electric fields and find the average lateral extent of the electron (hole) wave function to be 5 (3) nm. ${ }^{16}$ Using these values, we estimate $\beta=-4 \mu \mathrm{eV} /(\mathrm{kV} / \mathrm{cm})^{2}$ and for a Stark shift of $1 \mathrm{meV}, F=15 \mathrm{kV} / \mathrm{cm}$.

Figure 3 displays the Stark shift as a function of laser power for four biases on a different QD than that in Fig. 2 but in the same region of the device. As the laser power is increased, $F$ is reduced due to a space-charge effect. This can be explained by charge screening at the free surface; excited electrons and holes in the depletion region are swept in opposite directions and screen $F$. Figure 4 shows the intensity decrease due to $F$ for the neutral exciton presented in Fig. 1. This result is caused by a combination of reduced oscillator strength and an increased carrier tunneling probability. In addition, carriers excited at $850 \mathrm{~nm}$ must relax into the ground state. Carriers in the $p$ shell have an even larger tun-

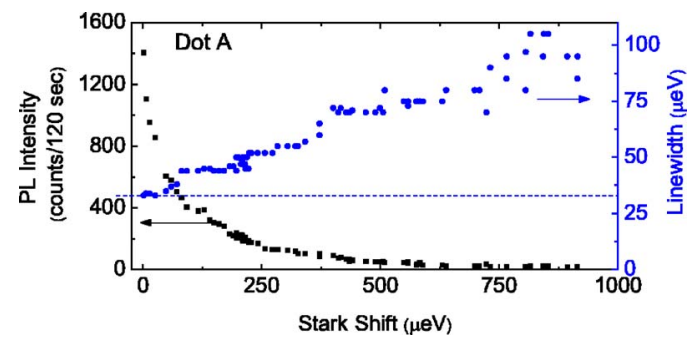

FIG. 4. (Color online) PL intensity and linewidth as a function of Stark shift. With an increasing electric field, the linewidth increases and a concurrent decrease in PL intensity is observed. The dashed line corresponds to the resolution limit of the spectrometer. 


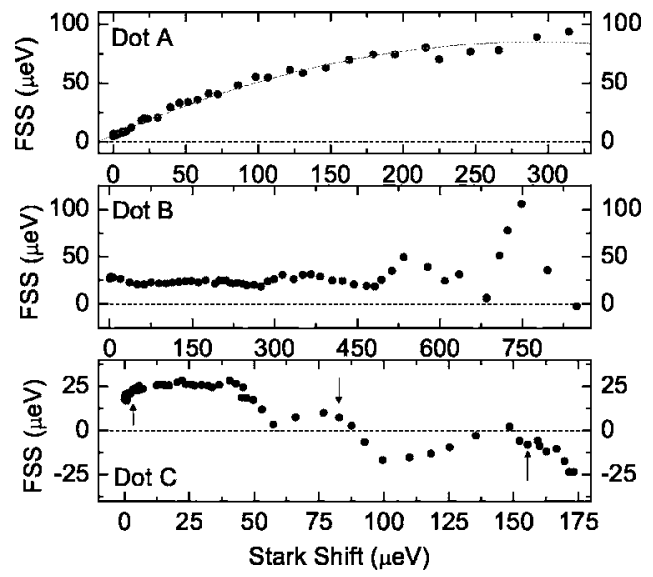

FIG. 5. FSS of three QDs as a function of Stark shift. In dot A, the splitting shows a square root dependence on the Stark shift. Dots B and C exhibit a splitting with an oscillatory response to the Stark shift. The arrows for dot C correspond to the values where the dipole orientations were measured.

neling probability than those in the $s$ shell due to $F$, effectively reducing the pumping rate into the ground state. The tunneling of carriers out of the ground state also leads to a reduced exciton lifetime and directly contributes to an increased linewidth. In addition, there may be spectral diffusion caused by the large fluctuation in charge at the nearby free surface. Due to significant line broadening observed at even a modest lateral $F$, the Stark shift could not be used as the modulation in transmission spectroscopy. ${ }^{5}$

Previous experiments on the QD FSS have found the linear polarization orientation to be orthogonal relative to the crystallographic fast growth direction $[1-10]{ }^{3,4,6,7,15} \mathrm{We}$ studied several QDs in the depletion region of our device and surprisingly find that the polarization orientation with respect to the crystal axis is not identical for each QD, similar to that reported for CdTe/ZnTe QDs. ${ }^{17}$ Nevertheless, values for the FSS between 5 and $30 \mu \mathrm{eV}$ at zero applied bias were observed. Figure 5 displays the FSS for three QDs as a function of Stark shift. The three QDs were located in the same sample in the same device and each had a PL energy near $953 \mathrm{~nm}( \pm 5 \mathrm{~nm})$ and therefore should be very similar. As the polarization orientation is not the same for each QD, the distinction of positive or negative for the FSS values is arbitrarily chosen.

In general, two types of behavior for the FSS as a function of $F$ are observed. For dot A in Fig. 5 the FSS increases with a square root dependence on the Stark shift. The intrinsic FSS value of $\sim 5 \mu \mathrm{eV}$ increases to $\sim 94 \mu \mathrm{eV}$ at the maximum $F$ for which there is still PL intensity. For QDs B and C the FSS shows evidence of an oscillatory response whereas the other properties of the QDs (i.e., linewidth, intensity, and energy) change monotonically with the Stark shift. In dot B the FSS approaches zero before increasing three times. Dot C shows evidence of oscillatory behavior in which the FSS remarkably crosses zero splitting. To identify if the dipoles are rotating in response to the electric field, polarization dependent PL was repeated at three different biases (identified by arrows in Fig. 5) for dot C. In each case, the dipole orientation was the same.

To engineer devices suitable for entangled photon production, these results indicate that a lateral electric field is sufficient to tune the FSS to zero. However, the strong effect of carrier tunneling is a considerable drawback. The nonra- diative process may affect the prospect of entanglement and inhibits experimental attempts to measure the photon statistics. Two strategies may circumvent this problem. One would be to find a QD with a small negative FSS that responds like dot A. In this scenario a very small electric field would be needed thus tunneling is unlikely. A second approach is to use QDs with a deeper confining potential such that the effect of carrier tunneling is reduced, although in such dots the initial FSS may be larger.

In summary, we have studied QDs in the depletion region of a Schottky gate. A lateral electric field was used to induce Stark shifts of up to $1.5 \mathrm{meV}$ in the ground state neutral exciton. Additionally, decreased PL intensity and linewidth broadening were observed due to the electric field. We report the manipulation of the FSS over a large range $(\sim 100 \mu \mathrm{eV})$ and most importantly through zero. Hopefully, these results stimulate a microscopic understanding of the electric field dependence on the exciton FSS.

The authors acknowledge financial support for this work from SANDIE (EU), EPSRC (UK), (ES) MEC, and CAM (TEC-2005-05781-C03-01, NAN2004-09109-C04-001, QOIT CSD2006-0019, and S-505/ESP/000200) and DAAD (DE).

${ }^{1}$ D. Bouwmeester, A. K. Ekert, and A. Zeillinger, The Physics of Quantum Information (Springer, Berlin, 2000).

${ }^{2}$ O. Benson, C. Santori, M. Pelton, and Y. Yamamoto, Phys. Rev. Lett. 84, 2513 (2000).

${ }^{3}$ C. Santori, D. Fattal, M. Pelton, G. S. Solomon, and Y. Yamamoto, Phys. Rev. B 66, 045308 (2002).

${ }^{4}$ R. M. Stevenson, R. M. Thompson, A. J. Shields, I. Farrer, B. E. Kardynal, D. A. Ritchie, and M. Pepper, Phys. Rev. B 66, 081302(R) (2002).

${ }^{5}$ A. Högele, S. Seidl, M. Kroner, K. Karrai, R. J. Warburton, B. D. Gerardot, and P. M. Petroff, Phys. Rev. Lett. 93, 217401 (2004).

${ }^{6}$ K. Kowalik, O. Krebs, A. Lemaitre, S. Laurent, P. Senellart, P. Voison, and J. A. Gaj, Appl. Phys. Lett. 86, 041907 (2005).

${ }^{7}$ R. M. Stevenson, R. J. Young, P. See, D. G. Gevaux, K. Cooper, P. Atkinson, I. Farrer, D. A. Ritchie, and A. J. Shields, Phys. Rev. B 73, 033306 (2006).

${ }^{8}$ S. Seidl, M. Kroner, A. Högele, K. Karrai, R. J. Warburton, A. Badolato, and P. M. Petroff, Appl. Phys. Lett. 88, 203113 (2006).

${ }^{9}$ R. Sequin, A. Schliwa, S. Rodt, K. Potschke, U. W. Pohl, and D. Bimberg, Phys. Rev. Lett. 95, 257402 (2006).

${ }^{10}$ A. I. Tartakovskii, M. N. Makhonin, I. R. Sellers, J. Cahill, A. D. Andreev, D. M. Whittaker, J. P. R. Wells, A. M. Fox, D. J. Mowbray, M. S. Skolnik, K. M. Groom, M. J. Steer, H. Y. Liu, and M. Hopkinson, Phys. Rev. B 70, 193303 (2004); W. Langbein, P. Borri, U. Woggon, V. Stavarache, D. Reuter, and A. D. Wieck, ibid. 69, 161301(R) (2004).

${ }^{11}$ N. Akopian, N. H. Lindner, E. Poem, Y. Berlatzky, J. Avron, D. Gershoni, B. D. Gerardot, and P. M. Petroff, Phys. Rev. Lett. 96, 130501 (2006).

${ }^{12}$ R. J. Young, R. M. Stevenson, P. Atkinson, K. Cooper, D. A. Ritchie, and A. J. Shields, New J. Phys. 8, 29 (2006); R. M. Stevenson, R. J. Young, P. Atkinson, K. Cooper, D. A. Ritchie, and A. J. Shields, Nature (London) 439, 179 (2006).

${ }^{13}$ D. A. B. Miller, D. S. Chemla, T. C. Damen, A. C. Gossard, W. Wiegmann, T. H. Wood, and C. A. Burrus, Phys. Rev. B 32, 1043 (1985).

${ }^{14}$ H. Gotoh, H. Kamada, H. Ando, and J. Temmyo, Appl. Phys. Lett. 76, 867 (2000); W. Heller, U. Bockelmann, and G. Abstreiter, Phys. Rev. B 57, 6270 (1998).

${ }^{15}$ M. Bayer, G. Ortner, O. Stern, A. Kuthar, A. A. Gorbunov, A. Forchel, P. Hawrylak, S. Fafard, K. Hinzer, T. L. Reinecke, S. N. Walck, J. P. Rathmaier, F. Klopf, and F. Schafer, Phys. Rev. B 65, 195315 (2002).

${ }^{16}$ R. J. Warburton, C. Schulhasuer, D. Haft, C. Schäflein, K. Karrai, J. M. Garcia, W. Schoenfeld, and P. M. Petroff, Phys. Rev. B 65, 113303 (2002).

${ }^{17}$ L. Marsal, L. Besombes, F. Tinjod, K. Kheng, A. Wasiela, B. Gilles, J. L. Rouviere, and H. Mariette, J. Appl. Phys. 91, 4936 (2002). 